\title{
A Fast Implementation of the Octagon Abstract Domain on Graphics Hardware
}

\author{
Francesco Banterle and Roberto Giacobazzi \\ Dipartimento di Informatica \\ Università degli Studi di Verona \\ Strada Le Grazie 15, 37134 Verona, Italy \\ E-mail: frabante@gmail.com and roberto.giacobazzi@univr.it
}

\begin{abstract}
We propose an efficient implementation of the Octagon Abstract Domain (OAD) on Graphics Processing Unit (GPU) by exploiting stream processing to speed-up OAD computations. OAD is a relational numerical abstract domain which approximates invariants as conjunctions of constraints of the form $\pm x \pm y<=c$, where $x$ and $y$ are program variables and $c$ is a constant which can be an integer, rational or real. Since OAD computations are based on matrices, and basic matrix operators, they can be mapped easily on Graphics Hardware using texture and pixel shader in the form of a kernel that implements matrix operators. The main advantage of our implementation is that we can achieve sensible speed up by using a single GPU, for each OAD operation. This can be the basis for an efficient abstract program analyzer based on a mixed CPU-GPU architecture.

Keywords: Octagon Abstract Domain, General Processing on GPU, Parallel Computing, Abstract Interpretation, Static Program Analysis.
\end{abstract}

\section{Introduction}

The study of stream processing (computing and programming) is recently gaining interest as an alternative and efficient methodology for allowing parallel processing in many fields of computer science. The paradigm is essentially based on defining a set of compute-intensive operations (called kernels) which are applied to each element in the stream. The growing success of this technology is related with the impressive grow in computational power of dedicated stream processing units (e.g., for graphic processing and more in general for digital signal processing) and in their relatively cheap costs. Recently researchers have become interested in developing algorithms for GPUs. These algorithms were at the beginning designed only for Computer Graphics purposes, but the high computational power offered pushed researchers to explore the possibilities of using GPU in more general tasks, leading to the so called General Purpose Computing on GPU (GP-GPU). GPUs were applied with success in various fields: Database [10], numerical methods $[2,14,9]$, and scientific computing $[11,8]$; see [21,23] for excellent surveys on GP-GPU.

In this paper we propose a new programming methodology to handle massive 
computations in numerical (relational) abstract domains. We consider stream processors and programming as an efficient and fast methodology for implementing the basic operators of abstract domains involving large matrices and massive data sets. Among the wide spectrum of numerical abstract domains, octagons [18] plays a key role due to their relational structure and affordable computational costs. Octagons provide a way to represent a system of simplified inequalities on the sum and difference of variable pairs, i.e. they represent constraints of the form $\pm x \pm y<=c$, where $x$ and $y$ are program variables and $c$ is a constant which can be an integer, rational or a real number automatically inferred. Their typical implementation is based on Difference Bound Matrices (DBM), a data structure used to represent constraints on differences of pairs of variables. Efficiency is a key aspect in this implementation: The space is constrained for $n$-variables in $O\left(n^{2}\right)$ and time is constrained in up to $O\left(n^{3}\right)$. This makes the relational analysis based on octagons applicable to large scale programs, e.g., those considered in the AstrÉE static analyzer [5] which employs programs having more than 10.000 global variables, most of them floating-point, in long-time iterations (about $3.6 \times 10^{6}$ iterations of a single loop). We prove that an important speed-up factor can be obtained by handling DBM in a stream-like computation model. In particular we exploit the structure of Graphics Processing Unit or (GPU), also called Visual Processing Unit, for an efficient and fast implementation of the abstract domain of octagons, in particular for a fast implementation of the basic operations on DBM. GPUs provide a dedicated hardware architecture for graphics rendering by exploiting a highly parallel structure making graphic computations far more effective than typical CPUs for a wide range of complex algorithms. This architecture is particularly suitable for operations on matrices, and therefore for handling operations on DBM. A typical GPU implements a number of graphics primitive operations in a way that implements stream processing: First, the data is gathered into a stream from memory. The data is then operated upon by one or more kernels, where each kernel comprises several operations. Finally, the live data is scattered back to memory. The static analyzer designed in our implementation is based on a CPU which manages the control flow and the GPU which performs the basic operators on the domain. Octagons are represented as 2D textures and the basic operations on octagons are implemented as kernel operations on their fragments. These operations can be performed in parallel on the texture due to their independence and thanks to the high degree of parallelism of the SIMD architecture like GPUs. The pipeline of the analyzer is therefore as follows: each time the analyser reaches a program point, the corresponding instruction is decomposed into basic abstract operations on octagons. The GPU is then activated to perform the basic computations, leaving the result in the video memory. As a result of our implementation, we obtain a sensible speed-up of several orders for simple operators on octagons (i.e., intersection, union, assignment, test guard, widening), and a speed-up around 24 times for the basic operation of octagon closure, which is performed each time octagons have to be merged. The bottleneck in our system is given by the test 
guard operator. This is due to the SIMD architecture of GPU which requires the spreading of the computation along the whole texture.

\section{The Octagon Abstract Domain}

The octagon abstract domain introduced by Miné in [18] is a (weakly) relational abstract domain which provides an upper approximation of program invariants as conjunctions of constraints of the form $\pm x \pm y<=c$, where $x$ and $y$ are program variables and $c$ is a constant which can be an integer, rational or a real. This abstract domain fits between the less precise linear-time non-relational abstract domain of intervals and the exponential-time relational approximation of convex polyhedra. Given a set of program variables $\mathcal{V}=\left\{V_{1}, \ldots, V_{n}\right\}$, we consider a set of enhanced variables: $\mathcal{V}^{\prime}=\left\{V_{1}^{\prime}, \ldots, V_{2 n}^{\prime}\right\}$ where for any $V_{i} \in \mathcal{V}$ we have both a positive form $V_{2 i-1}^{\prime}$, denoted $V_{i}^{+}$, and a negative form $V_{2 i}^{\prime}$, denoted $V_{i}^{-}$, in $\mathcal{V}^{\prime}$. A Difference Bound Matrix, or DBM for short, $\mathbf{m}$ is a $n \times n$ square matrix with elements in a field $\mathbb{Z}$ or $\mathbb{R}$ [18]. The element at line $i$ and column $j$, denoted $\mathbf{m}_{i j}$ equals a constant $c$ if there is a constraint of the form $V_{j}-V_{i} \leq c$, and $+\infty$ otherwise. Thus a conjunction of octagonal constraints in $\mathcal{V}$ can be represented as a DBM with $2 n$ dimension. In particular, a Galois connection has been established between DBM and sets of tuples of values:

$\gamma(\mathbf{m})=\left\{\left\langle v_{1}, \ldots, v_{2 n}\right\rangle \mid \forall i, j \leq 2 n . v_{j}-v_{i} \leq \mathbf{m}_{i j}\right\} \cap\left\{\left\langle v_{1}, \ldots, v_{2 n}\right\rangle \mid v_{2 i-1}=-v_{2 i}\right\}$

is the octagon represented by the $2 n$ dimension DBM $\mathbf{m}$. This $2 n$ space is isomorphic to a $n$-dimensional space which represent a convex structure having an octagon-like shape.

The set of (coherent) DBM, denoted cDBM, enriched with a bottom (empty) element $\perp^{\mathbf{c D B M}}$ representing the empty set $\varnothing$ and a top element $T^{\mathbf{c D B M}}$ representing the whole space, i.e., such that $\forall i, j . T_{i j}^{c \mathrm{cDBM}}=+\infty$, and ordered w.r.t. set inclusion, i.e., $\mathbf{m} \sqsubseteq \mathbf{n}$ iff $\forall i, j: \mathbf{m}_{i j} \leq \mathbf{n}_{i j}$, forms a complete lattice, where a DBM is coherent if $\forall i, j . \mathbf{m}_{i, j}=\mathbf{m}_{\overline{1} \bar{j}}$ where $\overline{\mathbf{1}}=$ if $i \bmod 2=0$ then $i-1$ else $i+1$. Intuitively a cDBM does not change by switching positive with negative forms of the same variable. The switch operation $\overline{1}$ is typically implemented by a bit-wise xor operation. The other classic lattice operators are defined as follows:

$$
\begin{aligned}
& \forall \mathbf{m}, \mathbf{n} \in \mathbf{c D B M} .\left(\mathbf{m} \sqcup^{\mathbf{c D B M}} \mathbf{n}\right)_{i j}=\max \left(\mathbf{m}_{i j}, \mathbf{n}_{i j}\right) \\
& \forall \mathbf{m}, \mathbf{n} \in \mathbf{c D B M} .\left(\mathbf{m} \sqcap^{\mathbf{c D B M}} \mathbf{n}\right)_{i j}=\min \left(\mathbf{m}_{i j}, \mathbf{n}_{i j}\right)
\end{aligned}
$$

The main result in the construction and representation of the octagon abstract domain is the existence of the best abstraction of octagons as an element in cDBM. This is achieved by computing normal forms for DBM representing octagons. A modified version of the Floyd-Warshall closure algorithm which performs strong closure is considered for this task. The intuition is that, while the Floyd-Warshall closure algorithm can be seen as a constraint propagation which completes a set of constraints until the following closure holds:

$$
\left\{\begin{array}{l}
V_{i}^{\prime}-V_{k}^{\prime} \leq a \\
V_{k}^{\prime}-V_{j}^{\prime} \leq b
\end{array} \Longrightarrow V_{i}^{\prime}-V_{j}^{\prime} \leq a+b\right.
$$




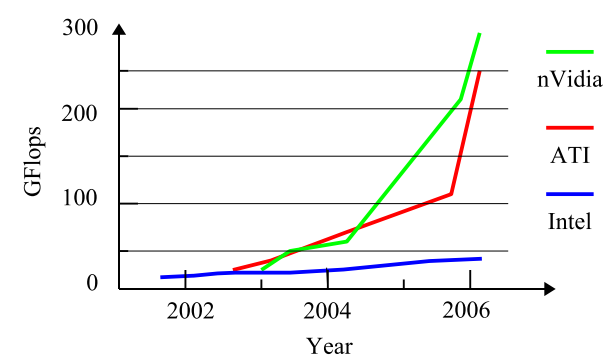

Fig. 1. A comparison of floating point performances between Intel CPU, ATI GPU and nVidia GPU in the last five years. As the graph shows GPU are increasing their performance every year faster than CPUs.

The modified Floyd-Warshall strong closure algorithm adds a second form of constraints until the following closure holds [18]:

$$
\left\{\begin{array}{l}
V_{\overline{1}}^{\prime}-V_{i}^{\prime} \leq a \\
V_{j}^{\prime}-V_{\overline{\mathrm{J}}}^{\prime} \leq b
\end{array} \Longrightarrow V_{j}^{\prime}-V_{i}^{\prime} \leq(a+b) / 2\right.
$$

The constraints introduced by the (strong) closure algorithm are called implicit in order to distinguish them from the explicit constraints considered to build the octagon. The strong closure operation on DBM is denoted $(\cdot)^{\bullet}$. All the standard lattice-theoretic operations and C-like transfer functions have been defined on cDBM in order to derive an abstract semantics which has been proved correct by abstract interpretation [18].

\section{An Overview on GPUs}

In the last few years graphics hardware, known as GPU, dramatically increased its computationally power and flexibility for answering the need to increase the realism in videogames and other graphics applications. GPUs are quite a cheap product and they offer high performances, for example in the case of nVidia GeForce7950-GX2 (a double GPU equipped with 512Mb of RAM) the cost is around 310 euro (March 2007) offering a peak of 384 GFlops with a 51.2 $\mathrm{Gb} / \mathrm{sec}$ bandwidth through the video memory, see figure Figure 2 for a complete sight on how a GPU is inserted in the traditional computer architecture. These performances are more than tripled compared with its predecessor the nVidia GeForce6800 Ultra, 58 GFlops with a $38.5 \mathrm{~Gb} / \mathrm{sec}$ bandwidth. Indeed GPUs have an average yearly rate of growth around 2.0, which actually is higher than Moore's Law growth rate for CPU, 1.4 per year. See Figure 1 for the trend of GFlops in GPU in the last years.

\subsection{The Programmable Graphics Pipeline}

GPUs are optimized to render a stream of geometric primitives (point, lines and triangles), called vertex buffer, onto an array of pixels called frame buffer. The GPUs became in the last years fully programmable for transforming and lighting vertices and pixels. The main purpose of GPU is to processes vertices and pixels. This processing follows the classic graphics pipeline, allowing in certain stages 


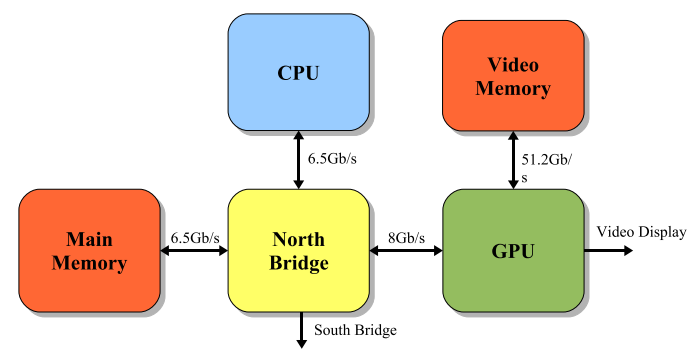

Fig. 2. The GPU in a traditional PC system: the GPU has a very large bandwidth with its memory (a peak of $51.2 \mathrm{~Gb} / \mathrm{s}$ in the nVida GeForce7900GTX). This value is nearly 8 times compared to the one for the CPU and its memory.

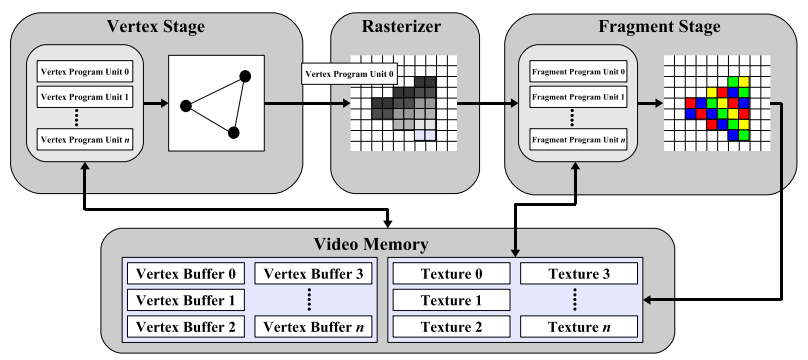

Fig. 3. The GPU Pipeline: vertices which define a primitive (triangle, point, line) are transformed using a function (implemented as a Vertex Program) in the Vertex Stage by Vertex Program Unit. Vertices are elaborated in parallel by many Vertex Program Units (Vertex Program Unit 0, ..., Vertex Program Unit $n$ ). Then primitives are discretized in fragments by the Rasterizer which passes these fragments to the Fragment Stage. At each fragment is applied a function (Fragment Program) in parallel using many Fragment Program Units (Fragment Program Unit 0, ..., Fragment Program Unit $n$ ). The result of the Fragment Stage is saved in a texture in the video memory.

programmability via micro programs. We can see a GPU pipeline in Figure 3. The first step in the pipeline is the vertices processing. Each vertex of a vertex buffer is processed by a Vertex Program Unit (VPU) which is a programmable unit that executes a vertex program (VP). A VP is a set of instructions that specifies how a vertex will be processed by the VPU. The output of a VPU is not only a modified position for a vertex but it can also add new properties to the vertex like a color, an address for fetching the memory during the next phase etc... A modern GPU presents more than one VPU (around 6) and it automatically distributes vertices to elaborate them fast between VPUs. Note that when a GPU is processing a single vertex buffer the VP is the same for all VPUs. After the VPU, a unit called rasterizer, generates fragments of the primitives, in other words it discretizes them in pixels and it interpolates values between vertices using linear interpolation. In the last step of the pipeline the fragments created by the rasterizer are processed by another programmable unit called Fragment Program Unit (FPU), which executes a fragment program (FP). As VP, the FP is a set of instructions which specifies how a fragment will be processed by the FPU. The output, which can be a single value or a vector of values, can be 
stored in the frame buffer for visualization or in a texture for future processing. As for VPUs, FPUs are numerous in a modern GPU (around 32), and fragments are automatically distributed to FPUs. Note that as for the VP, the same FP is executed by all FPUs until all fragments generated by rasterizer are completed. In this current generation of GPUs the main instructions allowed in the VPs and FPs are: float point operations (addition, subtraction, division, multiplication, square root, logarithm, etc...), random access to the video memory, assignment command, static and dynamic branching (a costly operation), and loop (with limited loop size for avoiding infinite loops). VPs and FPs are usually written in a high-level programming language similar to C. These languages are called shading languages because they are designed for generating images. The most common shading languages are: $\mathrm{C}$ for Graphics (Cg) [16], the OpenGL Shading Language (GLSL) [13] and High Level Shading Language (HLSL) [1]. These languages provide an abstraction for a very close level to the hardware, indeed they manage directly vertices, textures, fragments, etc... which are very specific graphics primitives. Other languages present an higher abstraction avoiding direct manipulation of graphics primitives and supporting GP-GPU such as SH [17], BrookGPU [3], and etc... The main disadvantage of these higher abstractions is that they are implemented on top of Graphics API such as OpenGL and Direct3D, so the overhead is quite high.

\subsection{Kernel Programming}

Data parallelism is the key for high performance in GPUs. In this section, we shortly introduce the GPU programming model called Kernel Model. The most powerful components in the GPU architecture are FPUs, because they are more numerous than VPUs, usually in a ratio 6:1, allowing more parallel power. A GPGPU program usually uses FPUs as main processing unit. The first algorithm is segmented into independent parallel parts, called kernels, each of these is implemented in a FPU. Inputs and outputs of each kernel are arrays of data, called texture, and they are stored in the video memory. A texture can be indexed in $1 \mathrm{D}$ (1D texture), in 2D (2D texture), and in 3D (3D texture). Note that $1 \mathrm{D}$ texture can have a size of only 4096 values, while $2 \mathrm{D}$ texture and 3D texture can allow a size up to respectively $4096^{2}$ and $512^{3}$ values. These are the following steps for kernel to run a kernel:

1. Vertices are passed to the GPU, in order to feed the vertex stage. A typical GP-GPU invocation is a quadrilateral, parallel to the screen of the display, which covers a region of pixels that match precisely with the desired size of the output texture. In our case this provides the extreme boundaries (a quadrilateral) of a temporary address space for allocating DBMs.

2. The rasterizer generates a fragment for every pixel in the quadrilateral. In our case the rasterizer fills the adress space with all the addresses, called fragments.

3. Each fragment is processed by the FPU. At this stage all the FPUs are processing the same fragment program. The fragment program can arbitrarily 


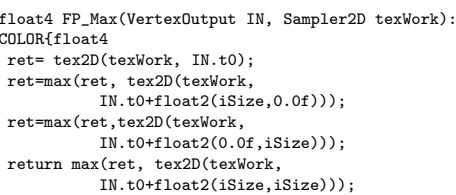

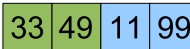

\begin{tabular}{l|l|l|l|}
61 & 5 & 21 & 3
\end{tabular}

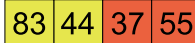

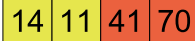

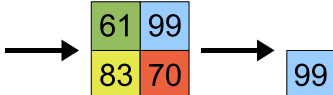

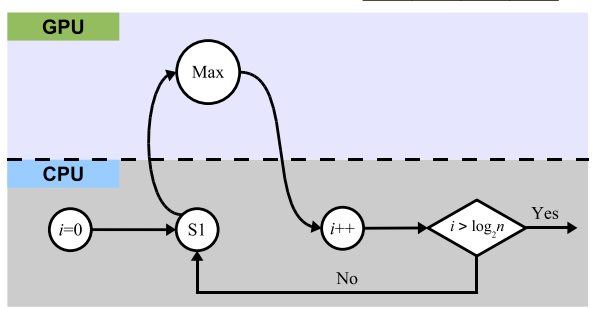

Fig. 4. The fragment program for calculating the maximum in a texture by reduction: IN is the input value of the FP calculated by interpolation from the rasterizer (IN.t0 is the vector that stores the coordinates of the current fragment), texWork is a $2 \mathrm{D}$ texture (declared as Sampler2D) in which are stored the values to reduce. The iSize is a constant value set in the FP, and represents the inverse of the size of the texture. tex $2 \mathrm{D}$ is required to access to a texture in the video memory. Below the control flow of the calculation of maximum value using reduction paradigm. First the CPU sets the counter $i=0$, then it enters in a loop. In the loop CPU transfers the control to GPU which calculates the maximum of every square of four pixels. After the GPU finishes it releases the control to CPU which increments $i$ and it tests $i>\log _{2}(n)$ where $n$ is the width of the texture. If the guard is true the reduction is completed otherwise the CPU returns in $\mathrm{S} 1$.

read from textures in the video memory, but can only write to memory corresponding to the location of the fragment in the frame buffer determined by the rasterizer. The domain of the computation is specified for each input texture by specifying texture coordinates at each of the input vertices of the quadrilateral. In our case, the fragment program computes locally to a single pixel the operations in the octagon domain.

4. The output of the fragment program is a value, that is stored in a texture.

An algorithm needs to reiterate this process as many times as required by the computation. This is called multi-pass.

\subsection{Reduction}

A constraint of current GPU's generation is that a FPU cannot randomly write the result of its job in the video memory, but only on an address which is chosen by the GPU's rasterizer. This is a problem if we want to compute properties from a texture such as maximum, or minimum value. The solution is a process called Reduction: the kernel program gets as input the value of four neighbor pixels and computes the needed function using these four values. At the end it saves the output in a texture which is reduced by one half. This process is iterated 
until the texture is only one single pixel; see Figure 4 for a visual example of the mechanism of reduction.

\subsection{Addresses Issues in GPUs}

In the current GPUs, integer arithmetic is not supported, only floating point is allowed. This feature will be present only in the upcoming GPU generation, R600 and GeForce Series 8 recently available. In particular when we want to access to a texture we need to use float addresses. The operator - ${ }^{-}$is used in the octagon domain to access to $v_{j}^{-}=-v_{j}$ the negative variable. This operator is defined as $i^{-} \stackrel{\text { def }}{=} i$ xor 1 . As we said before the XOR operation cannot be directly performed in the fragment. A solution to this problem is to encode in a single $1 \mathrm{D}$ texture, called XORTexture, all the XOR values for an address and save them in float values. Therefore, every time that we need to calculate a xor value of an address, we fetch the XORTexture with the address.

\subsection{Float Precision Issues}

GPUs can use two different types of floating point arithmetics: single precision, close to the 32-bit IEEE-754 standard, and half precision a 16-bit (10bits mantissa, 5bits exponent and 1bit sign). The main advantage of the half precision format is that a GPU performs nearly two times the speed of single precision, however they are not precise enough for numerical application, such as those employed in the octagon domain. We observed some numerical instabilities in the closure operation, therefore we decided to use the single precision format. This format has enough precision so there is no need to implement double precision in emulation using two single precision values (value and residual) [7]. GPUs present some issues with floating point arithmetic, because they do not implement the full IEEE-754 standard [12]. While GPUs can perform precise arithmetic operations (add, subtraction, multiplication, division, and tests) they cannot handle $\mathrm{NaN}$ value, and partially \pm Inf. Also isnan and isinf functions are very dependent by the drivers of the vendors which usually strongly suggest to avoid them in fragment programs. This can be a problem in order to represent $T^{\mathbf{c D B M}}=+\infty$ value in the octagon domain, however we simply solved this problem by assigning to $\top^{\mathbf{c D B M}}$ the value $3.4 e 38$, the maximum value representable in the GeForce series 6 and 7 architecture [22].

\section{Mapping Octagon Abstract Domain on GPU}

The Octagon Abstract Domain can be naturally mapped to GPUs because the data structure used to represent octagons, the DBM matrix, can be mapped one to one with a 2D texture of GPU. So there is no need to develop a particular data structure as in [15]. Another advantage is that the operators of the OAD are very simple matrix operators that can be performed by using simple kernel programming. In our implementation we did not use any API for GP-GPU. To 
avoid overheads, we directly wrote the code by using OpenGL and Cg language for writing fragment programs.

\subsection{Closure}

In the octagon domain closure represents the normal form for the DBM, which calculates all the constraints (implicit and explicit) between the variables. This operation is defined in [18] by the following algorithm, which is a modified version of the Floyd-Warshall shortest-path algorithm:

$$
\left\{\begin{array}{l}
\mathbf{m}_{0} \stackrel{\text { def }}{=} \mathbf{m}_{\text {def }} \\
\mathbf{m}_{k+1} \stackrel{\text { def }}{=} S\left(C_{2 k}\left(\mathbf{m}_{k}\right)\right) \quad \forall k: 0 \leq k \leq n \\
(\mathbf{m}) \stackrel{\text { def }}{=} \mathbf{m}_{n}
\end{array}\right.
$$

where $\mathbf{m}$ is an empty octagon, $C$ is defined as

$$
\left[C_{k}(\mathbf{n})\right]_{i j} \stackrel{\text { def }}{=}\left\{\begin{array}{rr}
0 & \text { for } i=j \\
\min \left(\mathbf{n}_{i j},\left(\mathbf{n}_{i k}+\mathbf{n}_{k j}\right),\right. & \\
\left(\mathbf{n}_{i \bar{k}}+\mathbf{n}_{\bar{k} j}\right), & \text { elsewhere } \\
\left(\mathbf{n}_{i k}+\mathbf{n}_{k \bar{k}}+\mathbf{n}_{\bar{k} j}\right), & \\
\left.\left(\mathbf{n}_{i \bar{k}}+\mathbf{n}_{\bar{k} k}+\mathbf{n}_{k j}\right)\right) &
\end{array}\right.
$$

and $S$ as

$$
[S(\mathbf{n})]_{i j} \stackrel{\text { def }}{=} \min \left(\mathbf{n}_{i j},\left(\mathbf{n}_{i \bar{i}} \mathbf{n}_{\bar{j} j}\right) / 2\right)
$$

This operation can be implemented on a GPU in the following way. First the

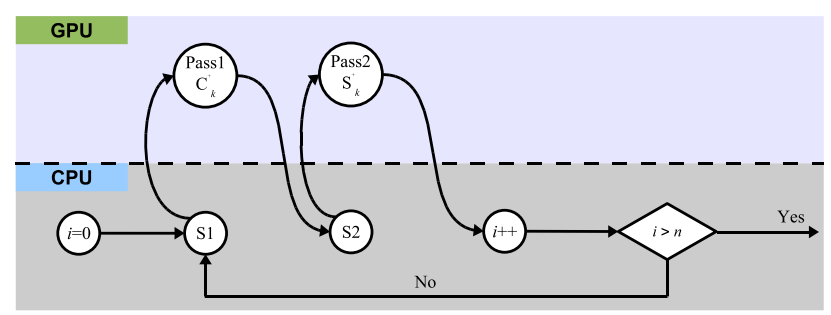

Fig. 5. The Closure control flow: at each cycle the CPU set parameters for the fragment program PS_Closure_C in S1 ( $\mathrm{k}$, and the working texture $T_{0}$ ), then the GPU executes PS_Closure_C saving the result in a texture, $T_{1}$. The CPU gets back the control and at stage S2 sets the parameter for fragment program PS_Closure_S (the working texture $T_{1}$ ), which is then executed by the GPU. Again the CPU gets back the control and increases the variable $i$. If $i>n$ (where $n$ is the number of variables in the program) the closure is reached, otherwise the CPU reiterates S1.

texture, $T_{0}$, representing the octagon that we want to close is applied $C$ using FP_CLOSURE_C in Figure 6, and it is saved in the video memory in another texture, $T_{1}$. Secondly at $T_{1}$ is applied $S$ using FP_CLOSURE_S in Figure 6 and the result is saved in $T_{0}$, see Figure 5 for a visualization of the control flow. This process is iterated $n$ times, where $n$ is the number of variables in the program. 


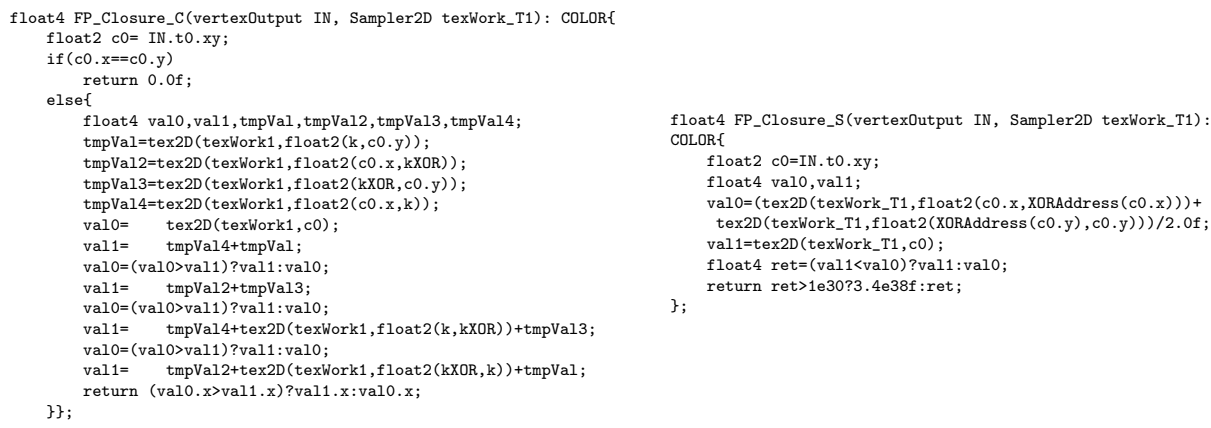

Fig. 6. FP_Closure_C is the FP that implements the $C$ function: values k and kXOR are constant values set by CPU, kXOR is the xorred value of $\mathrm{k} . \mathrm{k}$ represents the value $k$ in Equation 2. FP_CLOSURE_S implements $S$ (Equation 3).

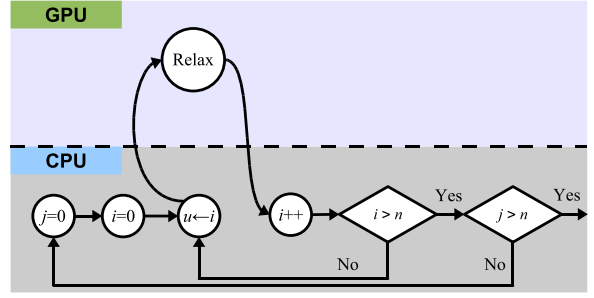

(a)

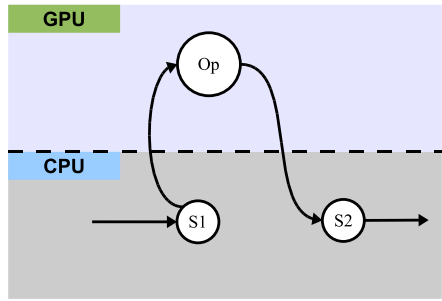

(b)

Fig. 7. GPU and CPU control flow: a) the control flow for the relaxation part of the Bellman-Ford Algorithm. b) the control flow of a simple operation, the CPU leaves the control to the GPU to execute the fragment program for the simple operation.

\subsection{Emptiness Test}

The emptiness test checks if an octagon $(\mathbf{m})^{\bullet}=\emptyset$. This happens if only if the graph of $(\mathbf{m})^{\bullet}$ has a cycle with a strictly negative weight [18]. A well known algorithm for detecting negative weight cycle is the Bellman-Ford algorithm. The mapping is realized as follow. Firstly we initialize texDist the distance array, a $1 \mathrm{D}$ Texture, using a constant shader which returns infinity (3.4e38). The size of this texture is $2 n$, where $n$ is the number of variables. Secondly we compute the first part of the algorithm, we iterate $(2 n)^{2}$ the FP_Relax, Figure 8, that relaxes edges, see Figure 7.a for control flow. Finally we call FP_Relax_Red, Figure 8, to find out if there is negative cycle. This function marks with value 1.0 a negative cycles, otherwise it return 0.0 . The result of the test is collected applying a reduction using a Maximum kernel Figure 4.

\subsection{Simple Operators}

There are some operators used in the octagon domain that require only one pass to obtain the result. This means that the fragment program for that operator is 


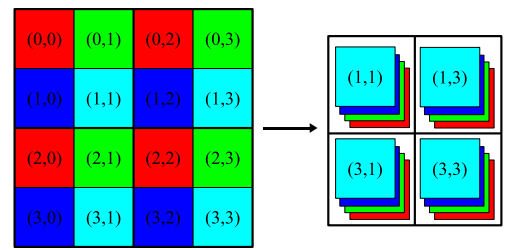

Fig. 12. The RGBA Packing: four neighbor values are packed in a single pixel using red, green, blue and alfa channels.

fragment programs, since GPU performs vector float point arithmetic operators, assignments, and the ternary operator (test?cond1:cond2). Also we do not use the values of a octagon in the dynamic branching (if-else), therefore we do not have to extend the branching for each components. Another common technique is to flatten $3 \mathrm{D}$ textures, this means to access to a $(i, j, k)$ memory location using a $(i, j)$ address. However representing octagon bigger than $8192 \times 8192$ using 3D texture implies to use more than $64 \mathrm{MB}$ of video memory for each, so we can keep on the GPU system only few octagons.

\subsection{Static Analyzer}

We designed a (naive) static analyzer as presented in $[6,18]$, in which the control flow is performed by the CPU but simple operators (union, intersection, assignments, guard checks), closure, widening, and fix point check are performed on GPU. When the interpreter starts to analyze a program, first it sets $\mathbf{m}=\top^{\mathbf{c D B M}}$ the octagon associated with the first program point, by using the simple fragment program in Figure 11. Then it interprets programs naively by applying octagon operators for the various commands and combinations of them:

$-\left[l_{i} v_{i} \leftarrow e l_{j}\right]:$ we used the assignment operators;

- $\left[l_{i}\right.$ if $g$ then $l_{j} \ldots$ else $l_{k} \ldots$ end if $\left.l_{p}\right]$ : for the branch $l_{j}$ we apply the guard operator $g$ to the octagon $\mathbf{m}_{i}$ ) (representing point $l_{i}$ ), while for the branch $l_{j}$ we apply the guard operator $\neg g$ to the octagon $\mathbf{m}_{i}$ ). When the flow control merges at point $l_{p}$ we use the union operator:

$$
\mathbf{m}_{p}=\left(\left(\mathbf{m}_{j}\right)^{\bullet}\right) \sqcup^{\mathbf{c D B M}}\left(\left(\mathbf{m}_{k}\right)^{\bullet}\right)
$$

- $\left[l_{i}\right.$ while $g$ do $l_{j} \ldots l_{k}$ done $\left.l_{k+1}\right]$ : we used the widening operator as presented in Section 4.4 for approximating the fixpoint.

\section{Results}

We implemented our abstract interpreter in $\mathrm{C}++$, by using OpenGL [20], a successful API for computer graphics that allows GPU programming, and $\mathrm{Cg}$ language for GPU programming. For results we used a machine equipped with an Intel Pentium 4 D 3.2 Ghz processor, 2GB of main memory, and a GeForce 7950-GTX with 512MB of video memory. We compared the results of our interpreter with a single threaded CPU interpreter. In our experiments, with randomly generated octagons, we timed single operators such as reduction, closure, 
intersection, union, assignment, test guard, widening, and emptiness test. The results for these operators by using a single GPU, compared with a CPU, are presented in Figure 13. For each operators we reached the following speed-ups,

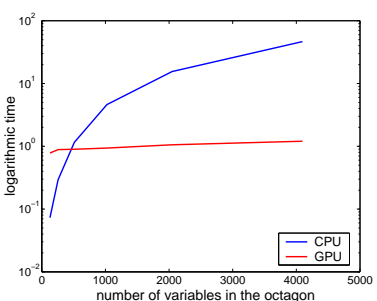

(a)

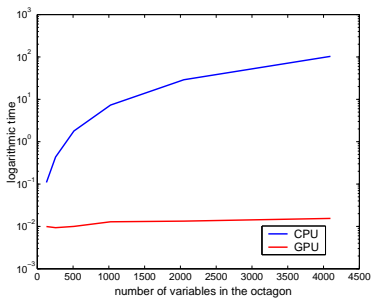

(c)

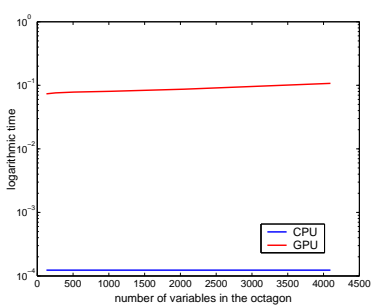

(e)

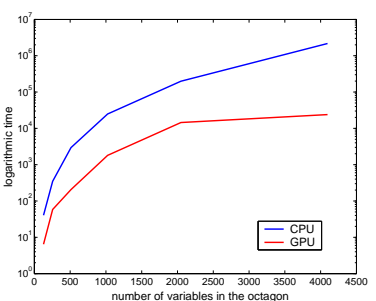

(b)

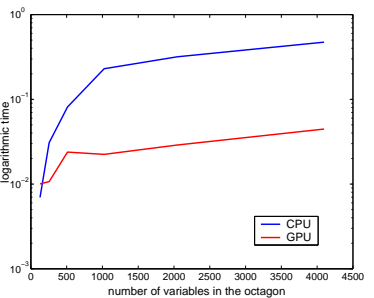

(d)

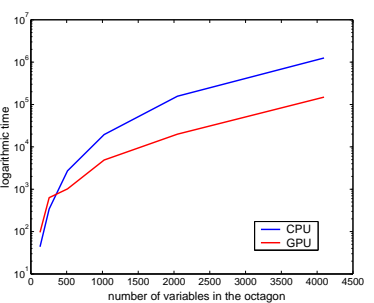

(f)

Fig. 13. Timing comparison between CPU and GPU for the octagon domain operators. We displayed timing in logarithmic scale of the time expressed in milliseconds: a) reduction operator (for checking fix point). b) closure operator. c) union (similar for intersection and widening). d) assignment. e) test guard. f) emptiness test.

that can be derived from Figure 13:

1. Reduction Operator: while the computational complexity for the CPU implementation is $\mathcal{O}\left(n^{2}\right)$, the one for GPU is $\mathcal{O}\left(\frac{n^{2}}{p} \log n^{2}\right)$, where $n$ is the number of variables in an octagon and $p$ is the number of FPUs. However its computational constant is lower than the one for CPU, so the speed-up is reasonable, achieving 9.98 times in average. 
2. Closure Operator: the computational complexity is the same for both implementations, GPU and CPU, $\mathcal{O}\left(n^{3}\right)$. We achieved a 24.13 times speedup in average.

3. Emptiness Test: the computational complexity is the same for both implementations, GPU and CPU, $\mathcal{O}\left(n^{3}\right)$. We achieved a 4.0 times speed-up in average. Note that the speed-up is lower than the one for the Closure Operator because we need to perform a test (FP_Relax_Red) and a reduction operation.

4. Union Operator: the computational complexity is the same for both implementations, GPU and $\mathrm{CPU}, \mathcal{O}\left(n^{2}\right)$. We achieved a 160.5 times speed-up in average.

5. Assignment Operator: while the computational complexity of this operator for the CPU implementation is $\mathcal{O}(n)$ (we need to modify only two columns), the one for GPU is $\mathcal{O}\left(n^{2}\right)$. This is caused by SIMD nature of GPUs that needs to work on all values of a texture and not only a portion of it. For this operator we reached a lower speed-up than other operators, 6.47 times in average.

6. Test Guard Operator: this represents the worst operator in our implementation test. Since for the CPU implementation we need to modify only a constant number of values in the octagon, its computational complexity is $\mathcal{O}(1)$. However on GPUs we cannot modify only few values in a texture, but all values, so the complexity is $\mathcal{O}(n)$. In this case the CPU performs better than GPU with a 175.43 times speed-up in average. However it is faster to perform this operator on GPU than a computation on CPU followed by a transfer to GPU. This is because we need to transfer a big amount of data through the bus, which is typically a bottleneck in the architecture (see Figure 2).

7. Widening Operator: as in the case of Union Operator, the computational complexity for both implementations is $\mathcal{O}\left(n^{2}\right)$. We achieved a 114.7705 times speed-up in average.

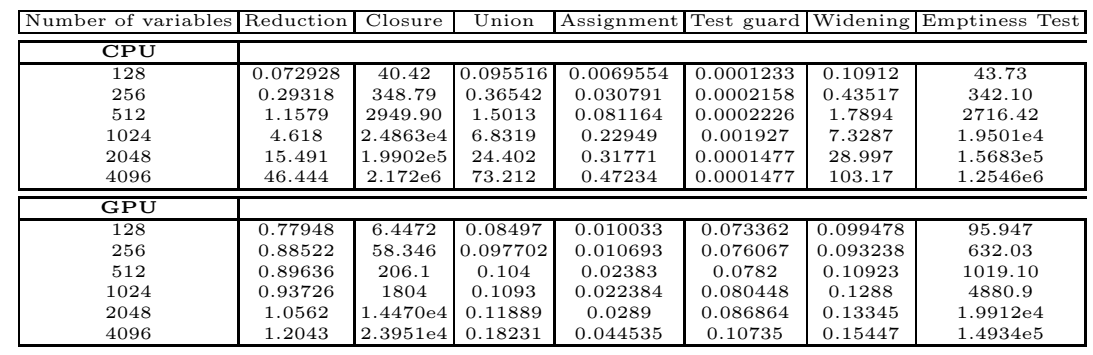

From the Table above the results of our implementation on CPU and GPU, the timing is expressed in second for 20 runs for each operator. As can we see, we obtain a sensible speed-up for simple operators (intersection, union, assignment, test guard, widening, emptiness test) and a speed-up around 24 times for closure operator. The bottleneck in our system is given by the test guard operator (Figure 13.e), indeed an optimized CPU implementation of this operator takes only $\mathcal{O}(1)$. 


\section{Conclusion and Future Work}

We presented a new implementation of Abstract Octagon Domain on GPU, improving efficiency in time. Another advantage of our implementation is that it is fully compatible with old GPUs (3-4 years models) and not only new models. Computational complexity of the algorithm is kept the same, with exception of the operation for checking if the fix point has been reached during the analysis of loops. While the complexity of this operation on CPU is $\mathcal{O}\left(n^{2}\right)$ where $n$ is the number of variables, it is $\mathcal{O}\left(\frac{n^{2}}{p} \log n^{2}\right)$, where $p$ is the number of FPUs, due to the overhead in the reduction phase. The main limits of our current implementation are: the test guard operator which is linear in the number of variables and the size of a DBM, which is $8192 \times 8192$, meaning that we can model octagons with 4096 variables using the RGBA packing technique. In future work we would like to extend the size of octagons using hierarchical techniques as presented in [15]. In these techniques, a larger texture is sliced in subtextures which are addressed using a page texture. A page texture presents as values pointers to access to the desired subtexture. We are also interested in upgrading our implementation with upcoming Graphics Hardware. One of the main advantages of the new generation is the ability to randomly write the results of a fragment program in the video memory. Therefore there will be no more need to perform reduction to check when to stop in the widening operator or for the emptiness test, improving the complexity and performance for these operators, which represents our main bottleneck. Another advantage would be the suppression of XORAddress function, since new GPUs present integer arithmetic, saving memory and GPU performance. The new generation presents a better floating point implementation (very close to IEEE754 standard, with still some issues for handling specials) that could improve our implementation. This improved precision does not solve the unsound problems, that can be solved using interval linear forms [19]. In future work we would like to map efficiently interval linear forms on GPU.

\section{References}

1. D. Blythe. The direct3d 10 system. In SIGGRAPH '06: ACM SIGGRAPH 2006 Papers, pages 724-734, New York, NY, USA, 2006. ACM Press.

2. J. Bolz, I. Farmer, E. Grinspun, and P. Schröoder. Sparse matrix solvers on the gpu: conjugate gradients and multigrid. ACM Trans. Graph., 22(3):917-924, 2003.

3. I. Buck, T. Foley, D. Horn, J. Sugerman, K. Fatahalian, M. Houston, and P. Hanrahan. Brook for gpus: stream computing on graphics hardware. ACM Trans. Graph., 23(3):777-786, 2004.

4. P. Cousot and R. Cousot. Abstract interpretation: a unified lattice model for static analysis of programs by construction or approximation of fixpoints. In Conference Record of the Fourth Annual ACM SIGPLAN-SIGACT Symposium on Principles of Programming Languages, pages 238-252, Los Angeles, California, 1977. ACM Press, New York, NY.

5. P. Cousot, R. Cousot, J. Feret, L. Mauborgne, A. Miné, D. Monniaux, and X. Rival. The ASTRÉE analyzer. In ESOP'05, volume 3444 of $L N C S$, pages 21-30. Springer, April 2005. 
6. P. Cousot and N. Halbwachs. Automatic discovery of linear restraints among variables of a program. In POPL '78: Proceedings of the 5th ACM SIGACTSIGPLAN symposium on Principles of programming languages, pages 84-96, New York, NY, USA, 1978. ACM Press.

7. G. Da Graçca and D. Defour. Implementation of float-float operators on graphics hardware. ArXiv Computer Science e-prints, March 2006.

8. Z. Fan, F. Qiu, A. Kaufman, and S. Yoakum-Stover. Gpu cluster for high performance computing. In SC '04: Proceedings of the 2004 ACM/IEEE conference on Supercomputing, page 47, Washington, DC, USA, 2004. IEEE Computer Society.

9. N. Galoppo, N. Govindaraju, M. Henson, and D. Manocha. Lu-gpu: Efficient algorithms for solving dense linear systems on graphics hardware. In $S C$ '05: Proceedings of the 2005 ACM/IEEE conference on Supercomputing, page 3, Washington, DC, USA, 2005. IEEE Computer Society.

10. N. Govindaraju, J. Gray, R. Kumar, and D. Manocha. Gputerasort: high performance graphics co-processor sorting for large database management. In SIGMOD '06: Proceedings of the 2006 ACM SIGMOD international conference on Management of data, pages 325-336, New York, NY, USA, 2006. ACM Press.

11. D. Reiter Horn, M. Houston, and P. Hanrahan. Clawhmmer: A streaming hmmersearch implementation. In SC '05: Proceedings of the 2005 ACM/IEEE conference on Supercomputing, page 11, Washington, DC, USA, 2005. IEEE Computer Society.

12. IEEE. Ieee 754: Standard for binary floating-point arithmetic.

13. J. Kessenich, D. Baldwin, and R. Rost. The opengl shading language v.1.20 revision 8. September 2006.

14. J. Kruger and R. Westermann. Linear algebra operators for gpu implementation of numerical algorithms. In SIGGRAPH '03: ACM SIGGRAPH 2003 Papers, pages 908-916, New York, NY, USA, 2003. ACM Press.

15. A. Lefohn, S. Sengupta, J. Kniss, R. Strzodka, and J. Owens. Glift: Generic, efficient, random-access gpu data structures. ACM Trans. Graph., 25(1):60-99, 2006.

16. W. Mark, R. Glanville, K. Akeley, and M. Kilgard. Cg: a system for programming graphics hardware in a c-like language. In SIGGRAPH '03: ACM SIGGRAPH 2003 Papers, pages 896-907, New York, NY, USA, 2003. ACM Press.

17. M. McCool, S. Du Toit, T. Popa, B. Chan, and K. Moule. Shader algebra. In SIGGRAPH '04: ACM SIGGRAPH 2004 Papers, pages 787-795, New York, NY, USA, 2004. ACM Press.

18. A. Miné. The octagon abstract domain. In AST 2001 in WCRE 2001, IEEE, pages 310-319. IEEE CS Press, October 2001.

19. A. Miné. Relational abstract domains for the detection of floating-point run-time errors. In ESOP'04, volume 2986 of $L N C S$, pages 3-17. Springer, 2004.

20. J. Neider and T. Davis. OpenGL Programming Guide: The Official Guide to Learning OpenGL, Release 1. Addison-Wesley Longman Publishing Co., Inc., Boston, MA, USA, 1993.

21. J. Owens, D. Luebke, N. Govindaraju, M. Harris, J. Krger, A. Lefohn, and T. Purcell. A survey of general-purpose computation on graphics hardware. In Eurographics 2005, State of the Art Reports, pages 21-51, August 2005.

22. M. Pharr and R. Fernando. GPU Gems 2 : Programming Techniques for HighPerformance Graphics and General-Purpose Computation. Addison-Wesley Professional, March 2005.

23. S. Venkatasubramanian. The graphics card as a stream computer. In SIGMODDIMACS Workshop on Management and Processing of Data Streams, 2003. 